[knowledge base]

Diamond Open Access

\title{
The membership relation
}

\author{
Open Mathematics Collaboration*†
}

March 17, 2021

\begin{abstract}
The MEMBERSHIP RELATION and its underlying definitions are presented in this white paper (knowledge base).
\end{abstract}

keywords: membership relation, abstract algebra, pure mathematics, knowledge base

The most updated version of this white paper is available at https://osf .io/r9p2m/download

*All authors with their affiliations appear at the end of this white paper.

†Corresponding author: mplobo@uft.edu.br|Open Mathematics Collaboration 


\section{Definition}

\section{MEMBERSHIP RELATION}

$$
\epsilon=\{(a, b) \in A \times A \mid a \in b\}
$$

$\epsilon, A, b:=$ sets

$\epsilon:=$ binary relation

$(a, b):=$ ordered pair

$A \times A:=$ Cartesian product

$(a, b \in A, a \in b) \rightarrow((a, b) \in \epsilon)$

$((a, b) \in \epsilon) \equiv(a \in b)$

$[1,2]$

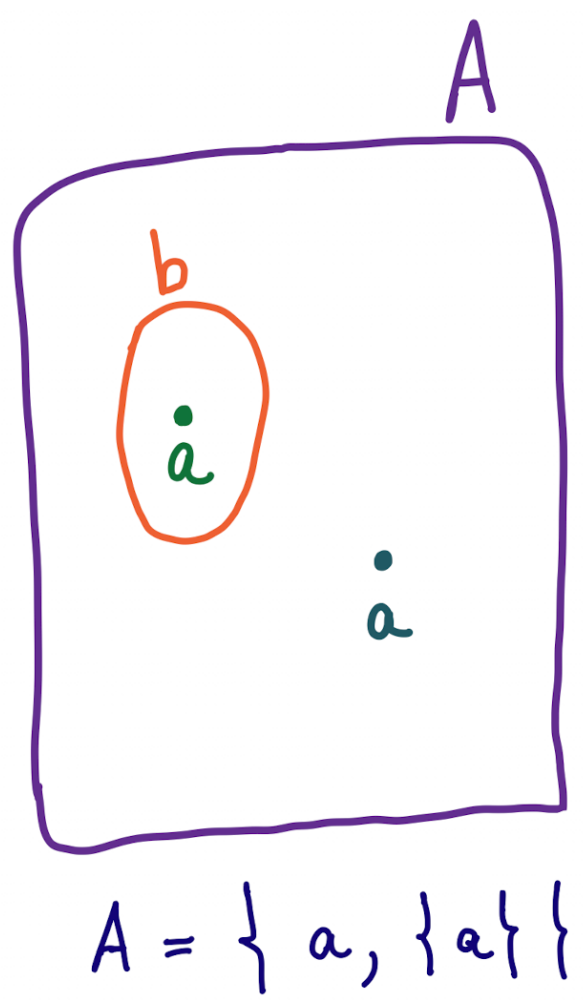

Figure 1: A diagram that illustrates the definition (1). 


\section{Example}

2. [1]

3. $A=\{\varnothing,\{\varnothing\}\}$

4. $A \times A=\{(\varnothing, \varnothing),(\varnothing,\{\varnothing\}),(\{\varnothing\}, \varnothing),(\{\varnothing\},\{\varnothing\})\}$

5. $\in \subset A \times A$

6. $\epsilon=\{(\varnothing,\{\varnothing\})\}$

7. $((\varnothing,\{\varnothing\}) \in \epsilon) \equiv(\varnothing \in\{\varnothing\})$

\section{Prerequisites}

8. Notation

$\subseteq:=$ subset relation

$c:=$ proper subset relation

[3]

9. Ordered pair

$$
(a, b)=\{\{a\},\{a, b\}\}
$$

$a:=$ first coordinate

$b:=$ second coordinate

$[1,3]$

10. Cartesian product

$$
A \times B=\{(a, b) \mid a \in A, b \in B\}
$$

$A, B:=$ sets

$A \times B:=$ Cartesian product

$(a, b):=$ ordered pair 
$[3]$

11. Binary relation on $A$

$$
R \subseteq A \times A
$$

$R, A:=$ sets

relation on $A:=$ binary relation on $A$

$(a, b) \in R \equiv a R b$

$[1,3]$

\section{Open Invitation}

Review, add content, and co-author this white white paper $[4,5]$.

Join the Open Mathematics Collaboration.

Send your contribution to mplobo@uft.edu.br.

\section{Open Science}

The latex file for this white paper together with other supplementary files are available in [6].

\section{Acknowledgements}

+ Center for Open Science https://cos.io

+ Open Science Framework https://osf .io 


\section{References}

[1] Warner, Steve. Abstract Algebra for Beginners. GET 800, 2018. https://books.google.com/books?id=UFleyAEACAAJ

[2] Warner, Steve. Pure Mathematics for Beginners. GET 800, 2018. https://books.google.com/books?vid=dcWrvAEACAAJ

[3] Velleman, Daniel J. How to prove it: A structured approach. Cambridge University Press, 2019.

https://books . google.com/books?vid=ISBN0521861241

[4] Lobo, Matheus P. "Microarticles." OSF Preprints, 28 Oct. 2019. https://doi.org/10.31219/osf .io/ejrct

[5] Lobo, Matheus P. "Simple Guidelines for Authors: Open Journal of Mathematics and Physics." OSF Preprints, 15 Nov. 2019. https://doi.org/10.31219/osf .io/fk836

[6] Lobo, Matheus P. "Open Journal of Mathematics and Physics (OJMP)." OSF, 21 Apr. 2020.

https://doi.org/10.17605/osf .io/6hzyp https://osf.io/6hzyp/files

[7] Lobo, Matheus P. "The Membership Relation." OSF Preprints, 17 Mar. 2021. https://doi.org/10.31219/osf.io/r9p2m

\section{The Open Mathematics Collaboration}

Matheus Pereira Lobo (lead author, mplobo@uft.edu.br) $)^{1,2}$ https://orcid.org/0000-0003-4554-1372

${ }^{1}$ Federal University of Tocantins (Brazil)

${ }^{2}$ Universidade Aberta (UAb, Portugal) 